EGU2020-15961

https://doi.org/10.5194/egusphere-egu2020-15961

EGU General Assembly 2020

(c) Author(s) 2020. This work is distributed under

the Creative Commons Attribution 4.0 License.

\title{
Automatic quality control and quality control schema in the Observation to Archive
}

Brenner Silva ${ }^{1}$, Najmeh Kaffashzadeh ${ }^{2}$, Erik Nixdorf ${ }^{3}$, Sebastian Immoor ${ }^{1}$, Philipp Fischer ${ }^{1}$, Norbert Anselm ${ }^{1}$, Peter Gerchow ${ }^{1}$, Angela Schäfer ${ }^{1}$, Roland Koppe ${ }^{1}$, and the Computing and data center ${ }^{*}$

${ }^{1}$ Alfred-Wegener-Institute, Computing and data center, Germany (bsilva@awi.de)

${ }^{2}$ Forschungszentrum Juelich

${ }^{3}$ Helmholtz Centre for Environmental Research

*A full list of authors appears at the end of the abstract

The O2A (Observation to Archive) is a data-flow framework for heterogeneous sources, including multiple institutions and scales of Earth observation. In the O2A, once data transmission is set up, processes are executed to automatically ingest (i.e. collect and harmonize) and quality control data in near real-time. We consider a web-based sensor description application to support transmission and harmonization of observational time-series data. We also consider a product-oriented quality control, where a standardized and scalable approach should integrate the diversity of sensors connected to the framework. A review of literature and observation networks of marine and terrestrial environments is under construction to allow us, for example, to characterize quality tests in use for generic and specific applications. In addition, we use a standardized quality flag scheme to support both user and technical levels of information. In our outlook, a quality score should pair the quality flag to indicate the overall plausibility of each individual data value or to measure the flagging uncertainty. In this work, we present concepts under development and give insights into the data ingest and quality control currently operating within the O2A framework.

Computing and data center: Stephan Frickenhaus (head, stephan.frickenhaus@awi.de) 